Original Article

\title{
ORAL ANTICOAGULANT PRESCRIPTION ANALYSIS FOR UTILISATION PATTERN AMONG IN- PATIENTS AT A TERTIARY CARE HOSPITAL
}

\author{
RESHMA ELSA JENY*, JENNY SUSAN SABU, MAMATHA KRISHNA MURTHY, NAGAMALESH UDIGALA MADAPPA \\ Department of Pharmacy Practice, M. S. Ramaiah College of Pharmacy, Rajiv Gandhi University of Health Sciences, M. S. R. I. T Post \\ Bengaluru 560054 Karnataka, India \\ Email: reshma.rej@gmail.com
}

Received: 03 Dec 2017 Revised and Accepted: 11 Jan 2018

\begin{abstract}
Objective: Selection of oral anticoagulants (OACs) in patients with thromboembolic diseases is complex and equivocal in nature, owing majorly to the non-adherence to standard clinical guidelines. Moreover, there is a scarcity of data on utilisation pattern of OACs in Indian population. Our primary objective was to analyse the prescribing pattern of OACs among in-patients with thromboembolic disorders. The secondary objective was to determine the significant predictors that affect their utilisation pattern.
\end{abstract}

Methods: This prospective observational study examined the clinical data over 6 mo from in-patients on oral anticoagulation with warfarin and acenocoumarol. Patients were recruited into 2 study groups: coronary artery disease (CAD) and non-CAD to analyse the prescription pattern. Potential predictors of utilisation pattern were evaluated for significance with logistic regression analysis for CAD group.

Results: Prescription analysis highlights preference of acenocoumarol (72.4\%) over warfarin among 232 in-patients in the study. Lack of achievement of target international normalised ratio (INR) levels was noticed in $74.2 \%$ of the study population. $57.8 \%$ of cases belonged to CAD group (males 76.7\%; mean age 59.4 \pm 13.5 y). Valvular heart disease (VHD) was the significant predictor $(\mathrm{p}=0.001)$ of warfarin utilisation among CAD patients while male gender, heart failure (HF) and arrhythmia were significant for acenocoumarol.

Conclusion: Predominance of age $<60$ y and male gender was observed in the study. Acenocoumarol was preferred over warfarin in all the inpatient departments. VHD, male gender, HF and arrhythmia significantly affected the OAC utilisation.

Keywords: Prescribing pattern, Oral anticoagulants, International normalised ratio, Drug-drug interactions

(c) 2018 The Authors. Published by Innovare Academic Sciences Pvt Ltd. This is an open access article under the CC BY license (http://creativecommons.org/licenses/by/4.0/) DOI: http://dx.doi.org/10.22159/ijpps.2018v10i3.24102

\section{INTRODUCTION}

Anticoagulants (AC) are vital lifesaving drugs used to prevent as well as treat arterial, venous and intra-cardiac thromboembolic events [1] As per the Global burden of disease study in India, coronary artery disease (CAD) is the main cause of cardiovascular diseases (CVDs) accounting for $35 \%$ of the disease burden [2]. The pharmacotherapy with the oral anticoagulants (OACs) are recommended for long-term thromboprophylaxis in patients at high risk of thrombotic complications. Also, OACs are observed to reduce the risk of atrial fibrillation (AF) complicating to stroke up to two thirds [3].

In India, the vitamin $\mathrm{K}$ antagonists (VKAs; warfarin and acenocoumarol) are the most prevalent choices, in spite of their narrow therapeutic index and interindividual treatment response variability, majorly due to the ease developed by the physicians after years of its usage. Henceforth, the dosage tapering and individualization of dose are vital for VKAs $[4,5]$.

Therapeutic management of thromboembolic disorders is often based merely on the clinical judgement of a prescriber, regardless of the availability of standard guidelines. Furthermore, the interplay of comorbidities and several concomitant drugs (polypharmacy), affect the efficacy of anticoagulation to a great extent [2].

Under and over-anticoagulation are the two major complications associated with anticoagulant use, which may lead to the risk of thrombosis and haemorrhage respectively. The aim of OAC therapy is to maintain an international normalised ratio (INR) of 2-3, which provides the best balance between the prevention of thromboembolic events and the occurrence of bleeding complications [6]. Therefore, monitoring of coagulation parameters and drug levels is a major concern due to lack of laboratories with standardised measurement, especially in ambulatory patients on $\mathrm{AC}$ use.

The rationale for conducting this study is the scarcity of Indian data on the pattern of anticoagulant use, monitoring and achievement of target INR. Our study aimed to analyse the prescribing pattern of OACs in a South-Indian tertiary care hospital and evaluate the effect of various predictors on the drug utilisation.

\section{MATERIALS AND METHODS}

Study setting and data collection procedure

The study was conducted for a period of six months in the departments of cardiology, neurology and vascular surgery at a tertiary care hospital that caters health care services to patients in and around Bengaluru. It was a prospective observational study approved by the Institutional ethical committee ECR/215/Inst/Ker/2013. The study included patients of both gender and more than $18 \mathrm{y}$ of age, treated with one or more OACs (acenocoumarol and warfarin). Pregnant women, patients on haemodialysis and those on other anticoagulants were excluded.

The data were collected from medication chart, case sheets, patient's previous medical records and prescriptions, laboratory investigation report and medication history interview forms. The data were evaluated for the prescribing pattern of OACs and drug-drug interactions (DDIs). The patients were categorised into CAD and non-CAD group based on their diagnosis according to the International classification of diseases version-10 (ICD-10), using data acquired from clinical manifestations, ECG changes, Echocardiography/angiography, Doppler findings and other laboratory investigations [7]. The study also investigated the significant predictors of OAC utilisation based on the demographic and clinical characteristics in CAD group. The severity of DDIs was categorised as major, moderate and minor using Micromedex ${ }^{\circledR}$ Solutions 2016 Truven Health Analytics LLC. Interactions were also classified as pharmacokinetic and pharmacodynamics interactions.

\section{Statistical analysis}

IBM statistical package for the social science version 20.0 (SPSS software) was used to generate graphs and data analysis. 
Descriptive analysis (mean $\pm \mathrm{SD}$ ) was used to determine numerical variables in the study population. The chi-square test was used to analyse the association between different variables in CAD and non$\mathrm{CAD}$ groups. Univariate logistic regression analysis was used to define the predictors of utilisation pattern in the CAD group treated with warfarin or acenocoumarol, which were later confirmed using multivariate analysis. Variables with $\mathrm{p}<0.05$ were identified as a statistically significant predictor of OAC utilisation in CAD patients.

\section{RESULTS}

\section{Demographics and clinical characteristics}

Among 232 patients, 177 patients (76.3\%) were males and the majority $(\mathrm{N}=120 ; 51.7 \%)$ were under the age group of $<60 \mathrm{y}$ (58.6 \pm 15.8 y). $134(57.8 \%)$ patients were diagnosed with CAD and $98(42.3 \%)$ patients with other conditions, i.e., non-CAD. Patients in CAD group were older compared to the non-CAD group (59.5years vs. 57.6years; $p=0.041$ ). The median length of stay (LOS) in the nonCAD group was only marginally higher [8 vs. $7.5 \mathrm{~d}]$ than CAD. However, statistical significance in this regard was not observed ( $p$ $=0.681$ ) [Table-1]. In our study, only 49 (21.1\%) patients had a body mass index (BMI) within the normal range of $18.5-24.9 \mathrm{~kg} / \mathrm{m}^{2}$. On the other hand, $108(46.6 \%)$ and 75 (32.3\%) patients were obese and overweight respectively.

The pattern of comorbidities among the two study groups-CAD and non-CAD depicted hypertension $(61.6 \%)$ as the most common, followed by diabetes mellitus (DM; $47.8 \%$ ). Hypertension, DM and heart failure (HF) were significantly higher in CAD patients while only CVA was significantly higher in non-CAD patients [Table-2]. Valvular heart disease (VHD) and arrhythmia were not statistically associated with CAD. Among the 232 patients, 65 (28\%) had a previous history of Percutaneous Transluminal Coronary Angioplasty (PTCA) and $37(16 \%)$ had undergone Coronary Artery Bypass Grafting (CABG).

Table 1: Baseline characteristics of patients with CAD and non-CAD

\begin{tabular}{llll}
\hline Variables & Total N=232 (100\%) & CAD N=134(57.8\%) & Non-CAD N=98 (42.2\%) \\
\hline Gendera; $\mathrm{n}(\%)$ & $177(76.3)$ & $103(76.7)$ & $74(75.5)$ \\
- Male & $55(23.7)$ & $31(23.1)$ & $24(24.5)$ \\
- Female & $58.6( \pm 15.8)$ & $59.4( \pm 13.5)$ & $57.6( \pm 18.5)$ \\
Age; Mean $( \pm S D)^{b}$ & $120(51.7)$ & $71(53)$ & $49(50)$ \\
$\bullet<60^{\text {a }}$ & $112(48.3)$ & $63(47)$ & $49(50)$ \\
• $>60^{\text {a }}$ & $8(6-11)$ & $7.5(5-10)$ & $8(6-11)$ \\
Median LOSc; days & $84(36.2)$ & $58(43.3)$ & $26(26.5)$ \\
Past surgical history; & & & 0.041 \\
n (\%) & & & 0.787 \\
\hline
\end{tabular}

${ }^{*} \mathrm{p}<0.05$ is considered statistically significant (Chi-square test), Data are given as anumber (n) of patients with percentages (\%) in parentheses or $\mathrm{as},{ }^{\mathrm{b}}$ mean \pm standard deviation or as, ${ }^{\mathrm{C}}$ Median with the interquartile range in parentheses.

Abbreviations: CAD (Coronary Artery Disease); LOS (Length of Stay); Non-CAD (Non-Coronary Artery disease).

Table 2: Common comorbidities among CAD and non-CAD patients

\begin{tabular}{|c|c|c|c|c|}
\hline Comorbidities & Total $^{\mathrm{a}} \mathrm{n}=232(100 \%)$ & CAD $^{a} n=134(57.75 \%)$ & Non-CAD ${ }^{a}=98(42.24 \%)$ & p-Value* \\
\hline Hypertension & $143(61.6)$ & $87(65)$ & $56(57.1)$ & 0.034 \\
\hline DM & $111(47.8)$ & $61(45.5)$ & $50(51)$ & 0.000 \\
\hline Heart failure & $61(26.3)$ & $42(31.3)$ & $19(19.4)$ & 0.000 \\
\hline VHD & $22(9.5)$ & $20(14.9)$ & $2(2)$ & 0.294 \\
\hline Arrhythmia & $24(10.3)$ & $22(16.4)$ & $2(2)$ & 0.345 \\
\hline CVA & $17(7.3)$ & $5(3.7)$ & $12(12.2)$ & 0.001 \\
\hline
\end{tabular}

${ }^{*} \mathrm{p}<0.05$ considered statistically significant (Chi-square test), a Data are given as number (n) of patients with percentages (\%) in parentheses., Abbreviations: CVA (Cerebrovascular Accident); DM (Diabetes mellitus); VHD (Valvular Heart Disease).

The pattern of duration of OAC therapy depicts that the majority of the population $(n=119 ; 52.3 \%$ ) were treated for $5-10 \mathrm{~d}$ during their hospital stay and the mean duration of therapy with OACs was calculated to be $5.39 \pm 2.78$ d. $44 \%$ of patients were treated for $<5 \mathrm{~d}$ while a low proportion of $4.7 \%$ patients were administered with OACs for $>10$ days. However, the duration of therapy largely depends on the diagnosis of individual patients and disease severity.

\section{Utilisation pattern of OACs among CAD and non-CAD patients}

Acenocoumarol (n= 168;72.4\%) was preferred over warfarin $(\mathrm{N}=64 ; 27.6 \%)$ in our study scenario and in all the three departments under study [fig. 1]. Utilisation pattern of acenocoumarol was observed to be higher than warfarin in CAD (65.7\% vs. $34.3 \%)$ and non-CAD (81.6\% vs.18.3\%) patients.

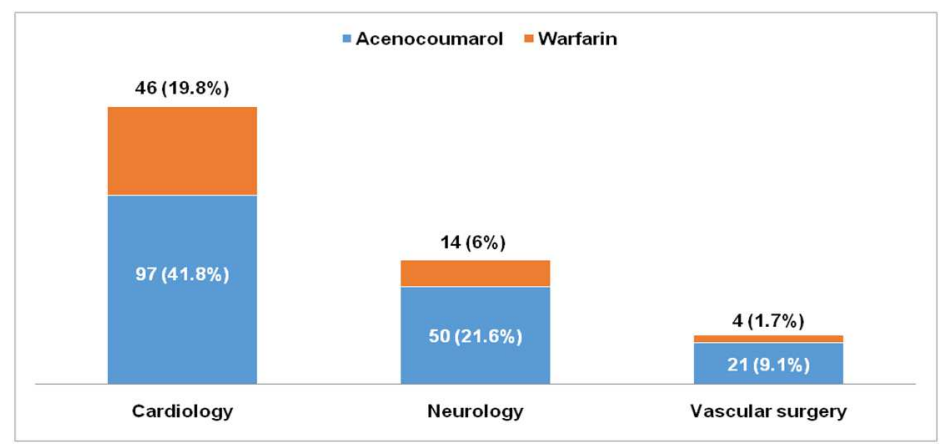

Fig. 1: Shows utilisation pattern of acenocoumarol and warfarin in each department under study 


\section{Determinants of utilisation of OACs in CAD patients}

Application of univariate analysis in patients on warfarin identified that age $<60$ y $[\mathrm{p}=0.042 ; 2.681(1.283-3.360)]$ and VHD $[\mathrm{p}=0.003$; $2.190(0.801-5.99)]$ were significant predictors of warfarin use. It was observed that patients with $<60$ y were 2.681 times more likely to be on warfarin than the elderly. Whereas, multivariate analysis established that VHD was the only significant predictor of warfarin which elevated its utilisation by 1.55 times $(\mathrm{p}=0.001)$. Male gender $(\mathrm{p}=0.038)$, HF $(\mathrm{p}=0.016)$ and arrhythmia $(\mathrm{p}=0.005)$ significantly increased the utilisation of acenocoumarol use by 1.61, 1.714 and 2.20 times respectively [table 3]. Multivariate analysis confirmed the significance of predictors for acenocoumarol use, derived as per the univariate analysis. Male gender $(\mathrm{p}=0.022), \operatorname{HF}(\mathrm{p}<0.0001)$ and arrhythmia $(\mathrm{p}=0.001)$ increased acenocoumarol utilisation correspondingly by $1.92,1.851$ and 1.71 times [table 4].

Table 3: Univariate analysis of warfarin and acenocoumarol utilisation pattern among CAD patients

\begin{tabular}{|c|c|c|c|c|c|c|}
\hline \multirow{2}{*}{$\begin{array}{l}\text { Variable } \\
\mathbf{N}\end{array}$} & \multicolumn{3}{|l|}{ Warfarin $^{b}$} & \multicolumn{3}{|c|}{ Acenocoumarolb $^{b}$} \\
\hline & n (\%) & p-Value & OR $(95 \% \mathrm{CI})^{\mathrm{a}}$ & n (\%) & p-Value & OR $(95 \% \text { CI) })^{a}$ \\
\hline Sex & & 0.48 & $1.370(0.572-3.283)$ & & 0.038 & $1.610(1.03-2.530)$ \\
\hline Male (103) & 37 (35.9) & & & $66(64)$ & & \\
\hline Female (31) & $9(29)$ & & & $22(21.4)$ & & \\
\hline Age & & 0.042 & $2.681(1.283-3.360)$ & & 0.188 & $0.614(0.298-1.268)$ \\
\hline$<60(71)$ & $27(38)$ & & & $44(62)$ & & \\
\hline$>60(63)$ & $19(30)$ & & & $44(70)$ & & \\
\hline Hypertension (87) & $32(36.8)$ & 0.234 & $0.626(0.289-1.354)$ & $55(63.2)$ & 0.32 & $1.598(0.789-3.459)$ \\
\hline Diabetes Mellitus (61) & $25(41)$ & 0.148 & $0.582(0.283-1.194)$ & $36(59)$ & 0.14 & $1.720(0.838-3.530)$ \\
\hline Heart Failure (42) & $19(45.2)$ & 0.162 & $0.586(0.274-1.242)$ & $23(54.8)$ & 0.016 & $1.714(0.805-3.649)$ \\
\hline VHD (20) & $12(60)$ & 0.003 & $2.190(0.801-5.99)$ & $8(40)$ & 0.142 & $4.558(1.670-2.441)$ \\
\hline Arrhythmia (22) & $4(18.2)$ & 0.448 & $1.481(0.537-4.087)$ & $18(81.8)$ & 0.005 & $2.20(1.21-4.01)$ \\
\hline CVA (5) & $3(60)$ & 0.241 & $0.776(0.125-4.820)$ & $2(40)$ & 0.786 & $1.288(0.207-7.995)$ \\
\hline
\end{tabular}

aOR (95\% CI): unadjusted odds' Ratio with 95\% confidence interval, bWarfarin: includes patients treated with warfarin; Acenocoumarol: includes patients treated with acenocoumarol, Data are given as number (n) of patients with percentages (\%) in parentheses, ${ }^{\mathrm{c}} \mathrm{p}<0.05$ is considered as statistically significant, Abbreviations: CVA (Cerebrovascular Accident); DM (Diabetes mellitus); VHD (Valvular Heart Disease).

Table 4: Multivariate analysis of warfarin and acenocoumarol utilisation pattern in CAD patients

\begin{tabular}{|c|c|c|c|c|}
\hline \multirow[t]{2}{*}{ Variable } & \multicolumn{2}{|l|}{ Warfarin $^{b}$} & \multicolumn{2}{|c|}{ Acenocoumarolb $^{\mathbf{b}}$} \\
\hline & p-Value & OR $(95 \% \mathrm{CI})^{\mathrm{a}}$ & p-Value & OR $\left(95 \%\right.$ CI) ${ }^{a}$ \\
\hline Sex & 0.693 & $1.245(0.420-3.694)$ & 0.022 & $1.92(1.402-2.52)$ \\
\hline Heart failure & 0.164 & $0.540(0.227-1.287)$ & $<0.0001$ & $1.851(0.777-4.411)$ \\
\hline VHD & 0.001 & $1.55(0.520-4.590)$ & 0.862 & $1.701(0.677-4.314)$ \\
\hline Arrhythmia & 0.135 & $2.835(0.722-11.131)$ & 0.001 & $1.71(1.30-1.81)$ \\
\hline
\end{tabular}

aOR (95\% CI): adjusted odds' Ratio with 95\% confidence interval, bWarfarin: includes patients treated with warfarin; Acenocoumarol: includes patients treated with acenocoumarol, ${ }^{\mathrm{c}} \mathrm{p}<0.05$ is considered as statistically significant, Abbreviations: VHD (Valvular Heart Disease).

\section{Monitoring pattern and achievement of target INR level}

It was found that most of the patients' INR was in sub-therapeutic range, i.e. $I N R<2(N=141 ; 60.8 \%)$. Merely, $4.7 \%(N=11)$ achieved target INR, while $13.4 \%$ of the overall patients attained supratherapeutic level (>3) [fig. 2]. The pattern of INR monitoring frequency indicates that $21.1 \%(n=49)$ of the study population was not advised for INR monitoring before initiation of VKA therapy.

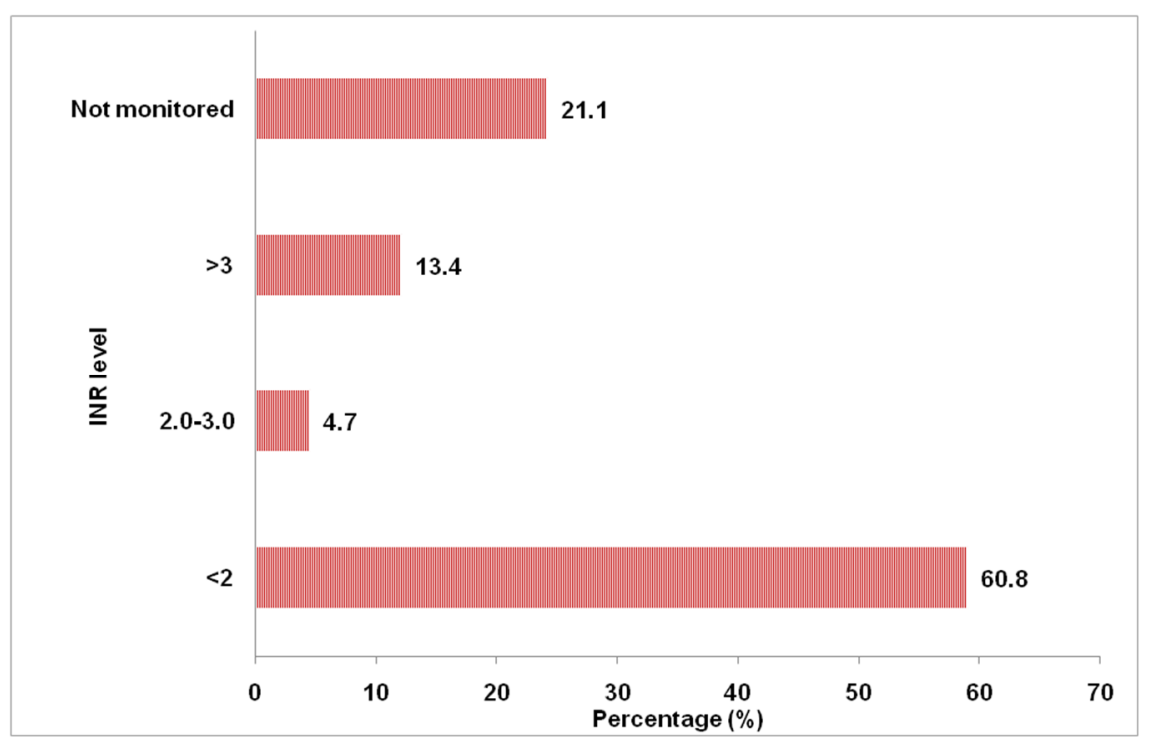

Fig. 2: Pattern of INR according to therapeutic range 
Fig. 2 shows the pattern of INR levels in the patients under study categorised according to therapeutic INR range (2-3). It also depicts the percentage of patients in whom INR monitoring was not adviced.

\section{Abbreviation: INR (International Normalised Ratio)}

\section{Prescription analysis for DDIs}

A total of 192 and 330 potential DDIs were found with warfarin and acenocoumarol respectively. The pattern of potential DDIs with warfarin revealed $39.6 \%$ of major interactions predominantly with aspirin (17.7\%). There were 25 (26\%) moderate interactions found with certain antibiotics such as azithromycin, cefoperazone etc. Also, $34.4 \%$ of minor interactions were observed with commonly used drugs like atorvastatin, furosemide, etc. There were $45.5 \%$ of major interactions observed with acenocoumarol, majorly with aspirin $(17.6 \%), 17 \%$ of moderate interactions were found with drugs like dexamethasone, esomeprazole etc. and minor interactions of $37.6 \%$ were also observed. There were $16.84 \%$ of pharmacokinetic and $46.4 \%$ of pharmacodynamic DDIs with acenocoumarol.

\section{DISCUSSION}

The pattern of hospital admissions for thromboembolic disorders in our study setting demonstrates a significant predominance of male gender and age $<60 \mathrm{y}$. Previous studies have reported an increased incidence of risk factors in this age group which reinforces that onset of cardiovascular disorders occurs at an early age [8,9]. Furthermore, epidemiological studies conducted in younger population indicate that the risk of developing cardiac disorders was higher in male $(49 \%)$ than in the female (32\%) group [10]. The majority of the patients were either obese or overweight $(78.9 \%$, $\mathrm{p}<0.0001$ ) which is analogous to a previous study [11]. This establishes the notion that obesity is a major predisposition for thromboembolic disorders [8].

WHO (2012) stated hypertension as the number one cause of mortality and a major risk factor for cardiac diseases and stroke in the worldwide [12]. Hypertension, a significant susceptibility factor, contributes to about $57 \%$ of $\mathrm{CAD}$ and $24 \%$ of stroke-induced mortality in India [13]. In addition, substantially elevated blood pressure levels have been observed in younger men than in women ( $27 \%$ vs. $12 \%$ ) [14]. It has also been known that hypertension, being a major contributor to thrombotic diseases such as DVT, increases the risk by twofold in the young male population [15]. Our study provides further evidence to these findings as hypertension was the most prevalent comorbid condition in the patients involved in our study, similar to the conclusions of other authors [2, 16, 17]. This prevalence of thromboembolic disorders could be indicative of the increased proportion of obesity, smoking or alcohol consumption and sedentary lifestyle among the younger males.

Our study presented that acenocoumarol was prescribed with a greater proportion of $72.4 \%$, which is in discordance with other South-Indian studies $[18,19]$. The differences in the usage of these drugs may be due to the prescribing preference among physicians in our study setting as acenocoumarol has been documented to provide steady anticoagulation and has well established a long-term safety profile, in spite of its short half-life [20]. However, controversies in the drug of choice indicate that comparative evaluation of these drugs should be validated in a larger cohort.

The evaluation of predictors for OAC utilisation among CAD patients found that VHD was the sole determinant of warfarin use. However, male gender, $\mathrm{HF}$ and arrhythmia were significant for acenocoumarol. The factors conducive to the preference of acenocoumarol in arrhythmia were the increased likelihood of bleeding and the higher incidence of a stroke upon treatment with warfarin. Several drug utilisation studies have contradicted the use of warfarin in patients with arrhythmia especially AF, due to these research outcomes and thereby indicate the inappropriateness of warfarin use for the pharmacological management of arrhythmic patients [21].

Acenocoumarol provides better stability of coagulation parameters in geriatrics, owing to their increased intrinsic sensitivity to OACs upon aging. Hence, prescribers do not prefer the use of warfarin in the elder population due to its relatively poor maintenance within the therapeutic INR range [22]. Warfarin utilisation was 2.681 times more preferred in age $<60 \mathrm{y}(\mathrm{p}=0.042)$. On the other hand, $70 \%$ of the elderly were treated with acenocoumarol, despite the lack of statistical significance.

In our study, only a mere percentage of 4.7 achieved these levels. The difficulty to maintain INR within the narrow therapeutic range has already been established. This may be due to its interactions with food and concomitant drugs, a dearth of patient cooperation and genetic causes, which further complicate the achievement of target INR levels [23]. In a study of warfarin utilisation, most of the patients $(73 \%)$ on OACs were within the therapeutic INR range of 23 [11]. The American College of Chest Physicians (ACCP) EvidenceBased Clinical Practice Guidelines-9 $9^{\text {th }}$ edition, suggest weekly INR testing in patients during initiation of VKA therapy [24]. In our study population, this stringent practice of weekly INR monitoring was observed in $21.2 \%$ of the cases which may be due to economic burden. This deficiency of INR monitoring should be addressed by physicians in India in order to prevent the incidence of bleeding among anticoagulated patients.

VKAs, due to their high plasma protein binding, CYP450 dependent metabolism and narrow therapeutic index escalate the potential risk of fatal interactions with concomitant drugs, prescribed for the management of various cardiovascular complications [25]. Nearly half of the study population had major DDIs, collectively for warfarin and acenocoumarol similar to other studies in Indian population. Pharmacodynamic interactions were estimated to be $70.9 \%$, which was only slightly higher than the estimates of Singh V et al. [26] $(65.7 \%)$. Furthermore, acenocoumarol was the most commonly identified drug with interactions, which is also parallel to our findings. However, its over-utilisation in our study population could have possibly contributed to this indictment against acenocoumarol.

The studied OACs were majorly interacting with antiplatelet drugs i.e., aspirin followed by clopidogrel which was similar to the prior studies $[25,27]$. Aspirin and several NSAIDs (Non-steroidal antiinflammatory drugs) exhibit their pharmacological actions via inhibition of Cyclo-oxygenase enzyme, thereby preventing the conversion of arachidonic acid to prostaglandins. Consequently, the formation of thromboxane $A_{2}$ in platelets is inhibited followed by prevention of thrombus formation. Upon concomitant administration of these drugs with anticoagulants, synergistic effect incline towards the increased haemorrhagic complications. Furthermore, pharmacokinetic DDIs occurs when such drugs compete for binding sites of warfarin and/or acenocoumarol [28]. The study also found DDIs with antibiotics such as azithromycin, cefoperazone etc. similar to Teklay G et al. [27]. Screening of drug interactions of OACs with concomitant medications is decisive, as it hinders the achievement of the therapeutic outcome.

\section{LIMITATION}

The study was carried out only for a short duration and was restricted to two oral anticoagulants-acenocoumarol and warfarin. One of the major limitations of our study was the failure to examine the confounding factors such as diet pattern, socioeconomic status, which could have influenced the significance of various factors on the utilisation pattern. The impact of OACs in patients with supratherapeutic INR levels could not be assessed due to the lack of feasibility of laboratory facilities for serum drug level analysis.

\section{CONCLUSION}

Acenocoumarol was preferred over warfarin, in all the three departments where the study was carried out. The predominance of male gender and age $<60 \mathrm{y}$ were observed among patients diagnosed with thromboembolic disorders. Our study highlights the need for achieving target INR levels and adherence to current guidelines with regard to the frequency of monitoring in anticoagulated patients. Maintenance of therapeutic INR levels is crucial for successful pharmacological management of thromboembolic disorders, preventing the occurrence of haemorrhagic events. Evaluation of determinants provides insight into the influence of patient-specific factors such as demographics and clinical comorbidities on the OAC utilisation pattern. 


\section{ACKNOWLEDGMENT}

We acknowledge Dr. V. Madhavan, Dean and Principal, Dr. E. Maheswari, Professor and Head of Department of Pharmacy Practice, Faculty of Pharmacy, M. S. Ramaiah University of Applied Sciences, Dr. Nagamalesh U. M, Consultant, Department of Cardiology, M. S. Ramaiah Hospitals for their enormous encouragement and guidance.

\section{FUNDING SOURCES}

This research did not receive any specific grant from funding agencies in the public, commercial, or not-for-profit sectors.

\section{DISCLOSURES}

The authors declare no conflict of interest. All the authors declare that they had no financial, personal relations towards other organisations whose interest could have affected the content of the article in either positive or negative way.

\section{AUTHORS CONTRIBUTIONS}

Dr. Reshma and Dr. Jenny wrote the primary draft of this paper and did the statistical analyses, which was edited by Mrs. Mamatha Krishna Murty for essential content. Dr. Nagamalesh helped with the review of the paper for a improved quality. All the authors reviewed and provided comments on further iterations.

\section{CONFLICT OF INTERESTS}

Declared none

\section{REFERENCES}

1. Anakwue RC, Ocheni S, Madu AJ. Utilisation of oral anticoagulation in a teaching hospital in Nigeria. Ann Med Health Sci Res 2014;4:286-90.

2. George J, Devi P, Kamath DY, Anthony N, Kunnoor NS, Sanil SS. Patterns and determinants of cardiovascular drug utilisation in coronary care unit patients of a tertiary care hospital. J Cardiovasc Dis Res 2013;4:214-21.

3. Association of Physicians in India. Oral Anticoagulants: Current Indian Scenario; 2013. Available from: http://www. apiindia.org/medicine_update_2013/chap90. [Last accessed on 02 Aug 2015]

4. Whitworth MM, Haase K, Fike DS, Bharadwaj RM, Young RB, MacLaughlin EJ. Utilisation and prescribing patterns of direct oral anticoagulants. Int J Gen Med 2017;10:87-94.

5. Chedepudi PR, Mounika O, Chandrika G, Franklin A, Ramesh M, Rani $\mathrm{V}$, et al. Impact of pharmacist-led anticoagulation monitoring and patient education on oral anticoagulation therapy with acenocoumarol. Asian J Pharm Clin Res 2017; 10:314-7.

6. Iuliano L, Matteo AD, Straface G. Management of nonvalvular atrial fibrillation: a comprehensive approach. Indian J Med Sci 2009;63:419-30.

7. WHO. International Classification of Diseases. Version 10; 2010. Available from: http://apps.who.int/classifications/ icd10/browse/2015/en/ [Last accessed on 05 Mar 2014]

8. Joshi P, Islam S, Pais P. Risk factors for early myocardial infarction in South Asians compared with individuals in other countries. JAMA 2007;297:286-94.

9. Bounda G, Ngarambe C, Ge WH, Yu F. Assessment and evaluation efficacy of a clinical pharmacist-led inpatient warfarin knowledge education program and follow-up at a Chinese tertiary referral teaching hospital. Arch Pharm Pract 2013;4:168-79.

10. Gomar FS, Quilis CP, Leischik R, Lucia A. Epidemiology of coronary heart disease and acute coronary syndrome. Ann Transl Med 2016;4:256-68.
11. Shehab A, Elnour A, Abdulle A, Souid AK. A prospective study on the use of warfarin in the United Arab Emirates. Open Cardiovasc Med J 2012;6:72-5.

12. Ma SM, John J. Assess the prevalence of hypertension and knowledge regarding the prevention of stroke. Asian J Pharm Clin Res 2017;10:177-80.

13. Anchala R, Kannuri NK, Pant H, Khan H, Franco OH, Di Angelantonio $\mathrm{E}$, et al. Hypertension in India: a systematic review and meta-analysis of prevalence, awareness, and control of hypertension. J Hypertens 2014;32:1170-7.

14. Everett B, Zajacova A. Gender differences in hypertension and hypertension awareness among young adults. Biodemography Soc Biol 2015;61:1-17.

15. Huang L, Li J, Jiang Y. Association between hypertension and deep vein thrombosis after orthopaedic surgery: a metaanalysis. Eur J Med Res 2016;21:1-7.

16. Lakshmi R, James E, Kirthivasan R. Study on the impact of clinical pharmacist's interventions in the optimal use of oral anticoagulants in stroke patients.Indian J Pharm Sci 2013;75:53-9.

17. Woods EA, Ackman ML, Graham MM, Koshman SL, Boswell RM, Barry AR. Anticoagulant and antiplatelet prescribing patterns for patients with atrial fibrillation after the percutaneous coronary intervention. Can J Hosp Pharm 2016;69:280-5.

18. Nekkanti H, Mateti UV, Vilakkathala R, Rajakannan T, Mallaysamy S, Padmakumar R. Predictors of warfarin-induced bleeding in a South Indian cardiology unit. Perspect Clin Res 2012;3:22-5.

19. Dhanya PS, Nidheesh C, Kuriakose KM, Puthiyaveetil N. Pattern of oral anticoagulant use following prosthetic heart valve replacement: a prospective observational study. Indian J Thoracic Cardiovascular Surgery 2011;27:119-24.

20. Trailokya A, Hiremath JS, Sawhney JPS, Mishra YK, Kanhere V, Srinivasa R, et al. Acenocoumarol: a review of anticoagulant efficacy and safety. J Assoc Physicians India 2016;64:88-93.

21. Elseviers M, Andersen M, Benko R, Andersen M, Benko R, Bennie $\mathrm{M}$, et al. Utilisation research in the area of cardiovascular medicines. Drug Utilisation Research: Methods and Applications, Chichester: John Wiley and sons; 2016. p. 290-3.

22. Trailokya A, Bahulayan A. Acenocoumarol in the geriatric population. J Clin Exp Cardiology 2016;7:1-2.

23. Mark L, Dani G, Vendrey R, Paragh G, Katona A. Oral anticoagulant therapy and bleeding events with vitamin $\mathrm{k}$ antagonists in patients with atrial fibrillation in a Hungarian county hospital. Med Sci Monit 2015;21:518-25.

24. Holbrook A, Schulman S, Witt DM, Vandvik PO, Fish J, Kovacs $\mathrm{MJ}$, et al. Evidence-based management of anticoagulant therapy: antithrombotic therapy and prevention of thrombosis: american college of chest physicians evidence-based clinical practice guidelines ( $9^{\text {th }}$ Edition). Chest 2012;141(2 Suppl):e152S-e184S.

25. Urtāne I, Bandere D, Ansabergs J, Stokmane AS. Role of clinical pharmacist in anticoagulation management. Med Theory Practice 2012;18:189-92.

26. Singh V, Gopinath K, Behzadpour A, Meera NK. Anticoagulant utilisation evaluation in a tertiary care teaching hospital: an observational prospective study in medical in patients. Indian J Pharm Practice 2015;8:61-6.

27. Teklay G, Shiferaw N, Legesse B, Bekele ML. Drug-drug interactions in risk of bleeding among inpatients on warfarin therapy: a prospective observational study. Thromb J 2014;20:1-8.

28. Moore N, Pollack C, Butkerait P. Adverse drug reactions and drug-drug interactions with over-the-counter NSAIDs. Ther Clin Risk Manag 2015;11:1061-75. 